\title{
Effects of magnetic braking and tidal friction on hot Jupiters
}

\author{
Adrian J. Barker and Gordon I. Ogilvie \\ Department of Applied Mathematics and Theoretical Physics, University of Cambridge, \\ Centre for Mathematical Sciences, Wilberforce Road, Cambridge CB3 0WA, UK \\ email: ajb268@cam.ac.uk
}

\begin{abstract}
Tidal friction is thought to be important in determining the long-term spin-orbit evolution of short-period extrasolar planetary systems. Using a simple model of the orbit-averaged effects of tidal friction (Eggleton et al. 1998), we analyse the effects of the inclusion of stellar magnetic braking on the evolution of such systems. A phase-plane analysis of a simplified system of equations, including only the stellar tide together with a model of the braking torque proposed by Verbunt \& Zwaan (1981), is presented. The inclusion of stellar magnetic braking is found to be extremely important in determining the secular evolution of such systems, and its neglect results in a very different orbital history. We then show the results of numerical integrations of the full tidal evolution equations, using the misaligned spin and orbit of the XO-3 system as an example, to study the accuracy of simple timescale estimates of tidal evolution. We find that it is essential to consider coupled evolution of the orbit and the stellar spin in order to model the behaviour accurately. In addition, we find that for typical Hot Jupiters the stellar spin-orbit alignment timescale is of the same order as the inspiral time, which tells us that if a planet is observed to be aligned, then it probably formed coplanar. This reinforces the importance of Rossiter-McLaughlin effect observations in determining the degree of spin-orbit alignment in transiting systems.
\end{abstract}

Keywords. Planetary systems - stars: rotation - celestial mechanics - stars: XO-3

\section{Introduction}

Since the discovery of the first extrasolar planet around a solar-type star (Mayor \& Queloz 1995), observers have now detected $322 \dagger$ planets around stars outside the solar system. Many of these planets have roughly Jovian masses and orbit their host stars in orbits with semi-major axes less than $0.2 \mathrm{AU}$, the so-called "Hot Jupiters" (HJs). In both of the giant planet formation scenarios, core accretion and gravitational instability, we are unable to produce HJs in situ. We require the formation of close-in planets much further out ( $a \sim$ several AU) in the protoplanetary disc, before a migratory process that brings the planet in towards the star and to its present location (Lin, Bodenheimer \& Richardson 1996).

The formation of systems with giant planets can be thought of as occurring in two oversimplified stages (Juric \& Tremaine 2008). During stage 1 the cores of the giant planets are formed, they accrete gas and undergo migration, driven by the dynamical interaction between the planets and the gaseous protoplanetary disc (see Papaloizou et al. 2008 for a recent review). This stage lasts a few Myr until the gas dissipates, by which time a population of gas giants may exist. If these form sufficiently closely packed then stage 2 follows. This stage lasts from when the disc has dissipated and continues until the present, and primarily involves gravitational interactions and collisions between

$\dagger$ As of the day of this session, 4th Nov. 2008-see http://exoplanet.eu/ 
the planets. Recent studies into stage 2 (Juric \& Tremaine 2008; Chatterjee et al. 2008) have shown that this is a chaotic era, in which planet-planet scatterings force the ejection of all but a few $(\sim 2-3)$ planets from the system in a period of large-scale dynamical instability lasting $\leqslant 10^{8} \mathrm{yr}$. This mechanism can excite the eccentricities of the planets to levels required to explain observations.

Planet-planet scatterings tend also to excite the inclinations of the planets with respect to the initial symmetry plane of the system, potentially leading to observable consequences via the Rossiter-McLaughlin (RM) effect, which allows a measurement of $\lambda$, the sky-projected angle of misalignment of the stellar spin and the orbit (Winn 2008). Misaligned orbits are not predicted from stage 1 alone, so if $\lambda$ is measured to be appreciably nonzero in enough systems, then it could be seen as evidence for planet-planet scattering, or alternatively Kozai migration (see Fabrycky \& Tremaine 2007). This is because gas-disc migration does not seem able to excite orbital inclination (Lubow \& Ogilvie 2001; Cresswell et al. 2007). Alternatively, if observed planets are all found with $\lambda$ consistent with zero, this could rule out planet-planet scattering or Kozai migration as being of any importance. One important consideration is that at such close proximity to their parent stars, strong tidal interactions are expected to change $\lambda$ (actually the true spin-orbit misalignment angle $i=\arccos (\hat{\mathbf{\Omega}} \cdot \hat{\mathbf{h}}))$ over time. If tides can significantly change $\lambda$ since the time of formation, we may have difficulty in distinguishing between the possible HJ formation mechanisms of planet-planet scattering, Kozai migration and gas-disc migration. Below we approach the problem of studying the effects of tidal friction on such inclined orbits.

\section{Model of tidal friction \& magnetic braking}

The efficiency of tidal dissipation in a body is usually parametrised by a dimensionless quality factor $Q$, defined by:

$$
Q=2 \pi E_{0}(\oint-\dot{E} d t)^{-1},
$$

where $E_{0}$ is the maximum energy stored in an oscillation and the integral represents the energy dissipated over one oscillation period. We find it convenient to define $Q^{\prime}=\frac{3 Q}{2 k_{2}}$, where $k_{2}$ is the second-order potential Love number, since this combination appears together in the theory.

$Q^{\prime}$ is in principle a function of the tidal forcing frequency and the amplitude of the tidal disturbance, and is a result of complex dissipative processes in each body (Zahn 2008). Recent work has shown that $Q^{\prime}$ for solar-type stars depends in a highly erratic way on the tidal forcing frequency (Ogilvie \& Lin 2007). In light of the uncertainties involved in calculating $Q^{\prime}$, and the difficulty of calculating the evolution when $Q^{\prime}$ depends on tidal frequency, we adopt a simplified model based on an equilibrium tide with constant lag time, using the formulation of Eggleton et al. 1998. This formulation is beneficial because it can treat arbitrary orbital eccentricities and stellar and planetary obliquities. We refer the reader to Barker \& Ogilvie (2009) or Mardling \& Lin (2002) for the full set of equations used.

Observations of solar-type stars show that the mean stellar rotational velocity decreases with main-sequence age (Skumanich 1972), following the relation $\Omega \propto t^{-1 / 2}$. Magnetic braking by a magnetised outflowing wind has long been recognised as an important mechanism for the removal of angular momentum from rotating stars (Weber \& Davis 1967), and such a mechanism seems able to explain (on average) most of the observed stellar spin-down (Barnes 2003). 
Here we include the effects of magnetic braking in the tidal evolution equations, through the inclusion of the magnetic braking torque of Verbunt \& Zwaan (1981). This gives the following term into of the equation for $\dot{\boldsymbol{\Omega}}$ (Dobbs-Dixon et al. 2004):

$$
\dot{\omega}_{m b}=-\alpha_{m b} \Omega^{2} \Omega
$$

where $\alpha_{m b}=1.5 \times 10^{-14} \mathrm{yr}$, giving a braking timescale $\sim 2 \times 10^{11} \mathrm{yr}$ for the Sun.

\section{Analysis of the effects of magnetic braking on tidal evolution for a simplified system}

We study the effects of magnetic braking on a simplified system of a circular, coplanar orbit under the influence of only the stellar tide and magnetic braking. The following set of dimensionless equations can be derived (Barker \& Ogilvie 2009):

$$
\begin{aligned}
\frac{d \tilde{\Omega}}{d \tilde{t}} & =\tilde{n}^{4}\left(1-\frac{\tilde{\Omega}}{\tilde{n}}\right)-A \tilde{\Omega}^{3} \\
\frac{d \tilde{n}}{d \tilde{t}} & =3 \tilde{n}^{\frac{16}{3}}\left(1-\frac{\tilde{\Omega}}{\tilde{n}}\right),
\end{aligned}
$$

where we have normalised the stellar rotation $\Omega$ and orbital mean motion $n$ to the orbital frequency at the stellar surface, together with a factor $C$ (to some power). $C$ is the ratio of the orbital angular momentum of an orbit with semi-major axis equal to the stellar radius $R_{\star}$, of a mass $m_{p}$, with the spin angular momentum of an equally rapidly rotating star of radius $R_{\star}$, mass $m_{\star}$ and dimensionless radius of gyration $r_{g}$. The reduced mass is $\mu=\frac{m_{\star} m p}{m_{\star}+m_{p}} . C$ is important for classifying the stability of the equilibrium curve $\tilde{\Omega}=\tilde{n}$ in the absence of magnetic braking, and it can be shown that this equilibrium is stable if $\tilde{n} \leqslant 3^{-\frac{3}{4}}$ i.e. no more than a quarter of the total angular momentum can be in the form of spin angular momentum (Hut 1980). We have thus defined the following dimensionless quantities:

$$
\begin{aligned}
\tilde{\Omega} & =\Omega\left(\frac{R_{\star}^{3}}{G\left(m_{\star}+m_{p}\right)}\right)^{\frac{1}{2}} C^{-\frac{3}{4}}, \quad \tilde{n}=n\left(\frac{R_{\star}^{3}}{G\left(m_{\star}+m_{p}\right)}\right)^{\frac{1}{2}} C^{-\frac{3}{4}}, \quad C=\frac{\mu R_{\star}^{2}}{I_{\star}}=\frac{\mu}{r_{g}^{2} m_{\star}}, \\
\tilde{t} & =\sqrt{\frac{G\left(m_{\star}+m_{p}\right)}{R_{\star}^{3}}\left(\frac{9}{Q^{\prime}}\right)\left(\frac{m_{p}}{m_{\star}}\right) C^{\frac{13}{4}} t,} \\
A & =\alpha_{m b} \sqrt{\frac{G\left(m_{\star}+m_{p}\right)}{R_{\star}^{3}}}\left(\frac{Q^{\prime}}{9}\right)\left(\frac{m_{\star}}{m_{p}}\right)\left(\frac{\mu R_{\star}^{2}}{I_{\star}}\right)^{-\frac{7}{4}} \simeq 100\left(\frac{Q^{\prime}}{10^{6}}\right) .
\end{aligned}
$$

There is only one parameter $(A)$ that completely characterises the solution in the $(\tilde{\Omega}, \tilde{n})$-plane, and its value may be estimated as $A \simeq 100$ for a Jupiter-mass planet orbiting a Sun-like star undergoing magnetic braking (with standard $\alpha_{m b}$ and with $Q^{\prime}=$ $\left.10^{6}\right)$. The size of this term shows that in general magnetic braking dominates the stellar spin evolution. We plot some solutions on the $(\tilde{\Omega}, \tilde{n})$-plane, restricting ourselves to $0 \leqslant$ $\tilde{\Omega} \leqslant 10,0 \leqslant \tilde{n} \leqslant 10$. This represents the full range of orbits of the HJs, since $\tilde{n} \simeq 10$ corresponds to an orbital semi-major axis of $a \simeq 0.01 \mathrm{AU}$, and $\tilde{n} \simeq 0.1$ corresponds to $a \simeq 0.2 \mathrm{AU}$.

With the inclusion of magnetic braking (see Fig. 1), an initially rapidly rotating solartype star hosting a HJ for which $\tilde{\Omega} \geqslant \tilde{n}$, will spin down as a result of the magnetic torque. During this stage of spin-down the spin frequency of the star may temporarily equal the orbital frequency of its close-in planet, but the rate of angular momentum loss 

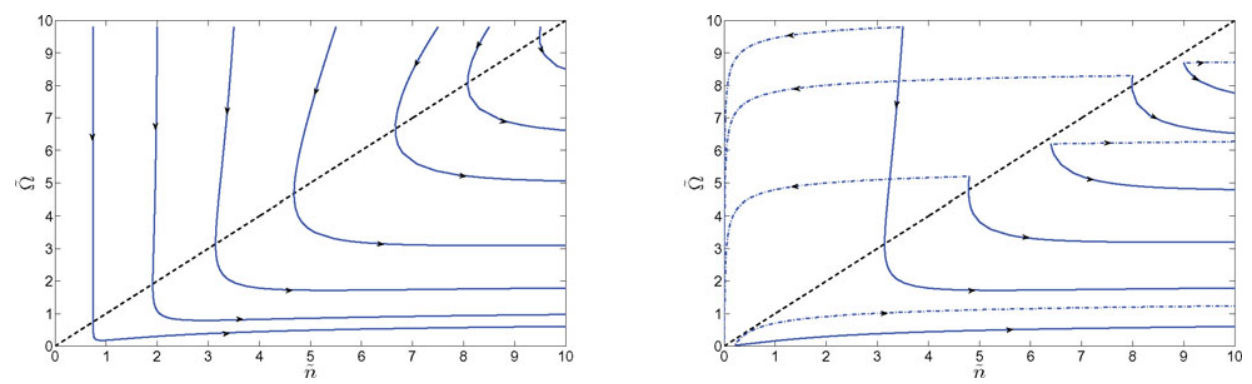

Figure 1. $(\tilde{\Omega}, \tilde{n})$-plane with $A=100$ for a HJ orbiting a Sun-like star. The dashed line in each plot corresponds to corotation $(\tilde{\Omega}=\tilde{n})$. Left: magnetic braking spins the star down so that the planet finds itself inside corotation, where the sign of the tidal torque changes, and the planet is subject to tidally induced orbital decay. Right: solutions with and without magnetic braking for the same initial conditions. Dot-dashed lines, which are also curves of constant total angular momentum, are without braking $(A=0)$ and solid lines are with braking $(A=100)$. This shows that the inclusion of magnetic braking is extremely important in determining the secular evolution of the system, and its absence results in a very different evolutionary history unless $\tilde{\Omega} \ll \tilde{n}$ in the initial state.

through magnetic braking will exceed the tidal rate of transfer of angular momentum from orbit to spin. The stellar spin continues to drop well below synchronism until the efficiency of tidal transfer of angular momentum from orbit to spin can compensate or overcompensate for the braking. The planet continues to spiral into the star once it moves inside corotation, and $\tilde{\Omega} \simeq$ const, unless the planet has sufficient angular momentum to be able to appreciably spin up the star. Note that any bound orbit will eventually decay in a finite time since the system has no stable equilibrium.

Without magnetic braking, the evolution is qualitatively different (see right plot Fig. 1), with orbits initially outside corotation $(\tilde{\Omega}>\tilde{n})$ asymptotically approaching a stable equilibrium $\tilde{\Omega}=\tilde{n}$ for $\tilde{n} \leqslant 3^{-\frac{3}{4}}$. Orbits initially inside corotation with $\frac{d \tilde{\Omega}}{d \tilde{t}}>\frac{d \tilde{n}}{d \tilde{t}}>0$ near corotation, also approach a stable equilibrium (though no such curves are plotted in Fig. 1, since they occur only in the far bottom left of the plot, near the origin). This is when the corotation radius moves inwards faster than the orbit shrinks due to tidal friction, resulting in a final stable equilibrium state for the system if the corotation radius "catches up" with the planet. For orbits inside corotation for which this condition is not satisfied, the planet will spiral into the star under the influence of tides.

\section{Tidal evolution timescales}

It is common practice to interpret the effects of tidal evolution in terms of simple timescale estimates. The idea behind these is that if the rate of change of a quantity $X$ is exponential, then $\dot{X} / X$ will be a constant, so we can define a timescale for the evolution of $X$, given by $\tau_{X}=X / \dot{X}$. If $\dot{X} / X \neq$ const, then $\tau_{X}$ may not accurately respresent the evolution. A tidal inspiral time can be calculated from $\tau_{a} \equiv-\frac{2}{13} \frac{a}{\dot{a}}$ (see Barker \& Ogilvie 2009 for the full evolution equations from which these timescales are derived), since $\frac{\dot{a}}{a} \sim a^{\frac{-13}{2}}$. This assumes $\Omega$ is constant, which we have seen is unreasonable unless $\Omega \ll n$, due to magnetic braking.

If the orbital and stellar equatorial planes are misaligned by a small angle $i$, then dissipation of the stellar tide would align them after a time

$$
\tau_{i} \equiv-\frac{i}{\frac{d i}{d t}} \simeq 35.0 \operatorname{Myr}\left(\frac{Q^{\prime}}{10^{6}}\right)\left(\frac{m_{\star}}{M_{\odot}}\right)\left(\frac{M_{J}}{m_{p}}\right)^{2}\left(\frac{R_{\odot}}{R_{\star}}\right)^{3}\left(\frac{P}{1 d}\right)^{4}\left(\frac{\Omega}{\Omega_{0}}\right)\left[1-\frac{P}{2 P_{\star}}\left(1-\frac{1}{\alpha}\right)\right]^{-1}
$$


where $\Omega_{0}=5.8 \times 10^{-6} \mathrm{~s}^{-1}$, and $P, P_{\star}$ are the orbital and stellar rotational periods. $\alpha$ is the ratio of orbit to spin angular momentum. The validity of these timescales to accurately represent tidal evolution is an important subject of study, since they are commonly applied to observed systems. Jackson et al. (2008) recently found it essential to consider the coupled evolution of $e$ and $a$ to accurately model tidal evolution, and that both the stellar and planetary tides must be included. They showed that the actual change of $e$ over time can be quite different from simple circularisation timescale considerations, due to the coupled evolution of $a$. In the following we consider the validity of $\tau_{i}$ to accurately model tidal evolution of $i$, using XO-3 as an illustrative example.

\section{Application to the misaligned spin and orbit of XO-3 b}

The only system currently observed with a spin-orbit misalignment is XO-3 (Hebrard et al. 2008), which has a sky-projected spin-orbit misalignment of $\lambda \simeq 70^{\circ} \pm 15^{\circ}$. This system has a very massive $m_{p}=12.5 M_{J}$ planet on a moderately eccentric $e=0.287$, $P=3.2 \mathrm{~d}$ orbit around an F-type star of mass $m_{\star}=1.3 M_{\odot}$. Its age is estimated to be $\tau_{\star} \simeq(2.4-3.1)$ Gyr. Note that even if the star is rotating near breakup velocity $\left(P_{\star} \sim 1\right.$ d), the planet is still subject to tidal inspiral, since $P_{\star}>P \cos i$ (where we henceforth assume $i=\lambda$, which may slightly underestimate $i$ ).

Hebrard et al. (2008) quote a spin-orbit alignment timescale of $\sim 10^{12} \mathrm{yr}$ for this system, but we find that this is in error by $\sim 10^{5}$. We believe that the reason for this discrepancy is that their estimate was based on assuming that the spin-orbit alignment time for XO-3 b is the same as for HD17156 b, which is a less massive planet on a much wider orbit. We find $\tau_{i} \sim 15$ Myr assuming $Q^{\prime}=10^{6}$ to align the whole star with the orbit. Integrations for this system are given in Fig. 2 for a variety of stellar $Q^{\prime}$ values. For the system to survive and remain with its current inclination for $\sim 3$ Gyr we require $Q^{\prime} \geqslant 10^{10}$. These integrations highlight the importance of considering coupled evolution of the orbital and rotational elements, since timescales for tidal evolution are quite different from simple estimates. Indeed, the actual spin-orbit alignment time from integrating the coupled equations is about an order of magnitude smaller than that from the simple decay estimate, due to coupled $a$ evolution.

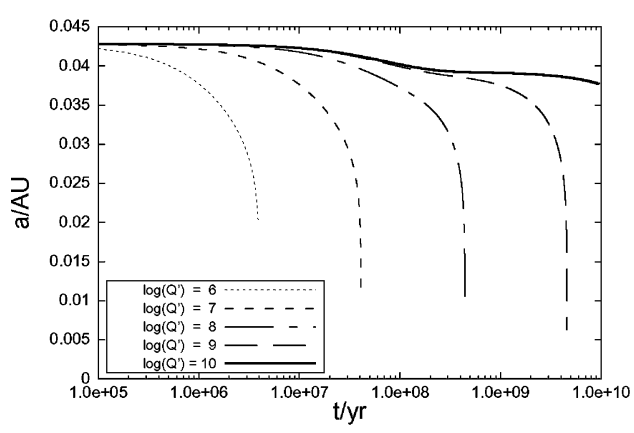

(a) semi-major axis

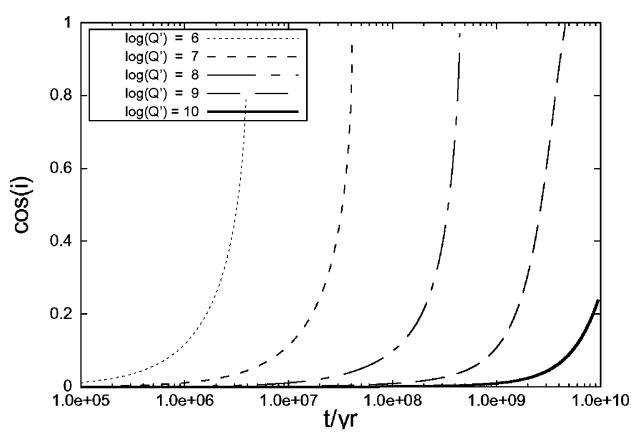

(b) cosine of inclination

Figure 2. Tidal evolution of XO-3 b taking current values for the orbital properties of the system, except that $\cos i=90^{\circ}$ (not unreasonable since this corresponds to the upper limit on $\lambda$, which in any case gives a lower bound on $i$ ). Magnetic braking is included, and $\Omega / n=2$ initially (results do not depend strongly on this choice). From (a) and (b) we require $Q^{\prime} \geqslant 10^{10}$ for the planet to survive for several Gyr, and maintain its high inclination. Tidal dissipation in star must therefore be weak to explain the current configuration of the system.

For this system, and for typical HJs, we find that $\tau_{i} \sim \tau_{a}$. This means that if we observe a planet, then its survival implies that tides are unlikely to have aligned its orbit. 
In addition, for systems on an accelerating inspiral into the star, the rate of inclination evolution will have been much lower in the past; therefore, if we observe a planet well inside corotation with a roughly coplanar orbit, we can assume that it must have started off similarly coplanar.

An explanation for the survival and remnant orbital inclination of XO-3 b could be the result of inefficient tidal dissipation in the host star. A calculation of $Q^{\prime}$ using the numerical method of Ogilvie \& Lin (2007) and a stellar model appropriate for this star was performed (Barker \& Ogilvie 2009). This predicts that the dissipation is weak, and $Q^{\prime} \geqslant 10^{10}$ for most tidal frequencies for the host star XO-3. This can explain the survival and remnant inclination of this planet, since both inspiral and spin-orbit alignment take longer than the current age of the system.

\section{Conclusions}

Magnetic braking is important for calculating the long-term tidal evolution of HJs unless $\Omega \ll n$, and changes the qualitative behaviour of the evolution significantly. Tidal evolution can be much faster than simple timescale estimates predict when coupled integration of the orbital and rotational elements is considered. In addition, we find that $\tau_{i} \sim \tau_{a}$ for typical HJs, so the orbits of most close-in planets have probably not aligned, and are likely to be a relic of the migration process. This means that RM effect observations of transiting planets can potentially distinguish between planet-planet scattering, Kozai migration and gas-disc migration.

\section{Acknowledgements}

A.J.B would like to thank the STFC for a research studentship.

\section{References}

Barker, A. J. \& Ogilvie, G. I. 2009, MNRAS, in preparation

Barnes, S. A. 2003, ApJ 586, 464

Chatterjee, S. et al. 2008, ApJ 686, 580

Cresswell, P. et al. 2007, A\& A 473, 329

Dobbs-Dixon, I., Lin, D. N. C., \& Mardling, R. A. 2004, ApJ 610, 464

Eggleton, P. P. and Kiseleva, L. G. and Hut, P. 1998, ApJ 499, 853

Fabrycky, D. and Tremaine, S., 2007, ApJ 669, 1298

Hebrard, G. et al. 2008, A\&A 488, 763

Hut, P. 1980, A\&A 92, 167

Hut, P. 1981, $A \& A$ A9, 126

Jackson, B., Greenberg, R., \& Barnes, R. 2008, ApJ 678, 1396

Jurić, M. \& Tremaine, S. 2008, ApJ 686, 603

Lin, D. N. C., Bodenheimer, P., \& Richardson, D. C. 1996, Nature 380, 606

Lubow, S. H. and Ogilvie, G. I. 2001, ApJ 560, 997

Mardling, R. A. \& Lin, D. N. C. 2002, ApJ 573, 829

Mayor, M. \& Queloz, D. 1995, Nature 378, 355

Ogilvie, G. I. \& Lin, D. N. C. 2007, ApJ 661, 1180

Ogilvie, G. I. \& Lin, D. N. C. 2004, ApJ 610, 477

Papaloizou, J. C. B. et al. 2007, Protostars and Planets V, 655

Skumanich, A. 1972, ApJ 171,565

Verbunt, F. \& Zwaan, C. 1981, A\&A 100, L7

Weber, E. J. \& Davis, L. J. 1967, ApJ 148, 217

Winn, J. N. 2008, ArXiv e-prints, 0807.4929

Zahn, J.-P. 2008, Tidal dissipation in binary systems, EAS Publications Series 29, 67 


\section{Discussion}

JARDINE: Do your results for the banal (orbital) fate of planets depend on the assumption that there is no outer planet forcing the orbit?

BARKER: Outer planets are not included in the integrations that I have performed thus far. Their inclusion may indeed change the final results and this should be studied for any system.

KHODACHENKO: What could possibly be the outcomes of the inclusion of electrodynamic effects of interaction of planetary dipole with the conducting plasma environment in your model? This may provide an additional energy dissipation channel and introduce an additional force into the system.

BARKER: An interesting question, and one that I am planning on studying in the next few years of my PhD. It would be interesting to study whether this $\mathbf{B}$ force could be significant enough to cause any orbital changes of the planet. In addition, dissipation of time-varying stellar magnetic-field excited currents in the planet could potentially inflate the planet. This is something that needs to be studied.

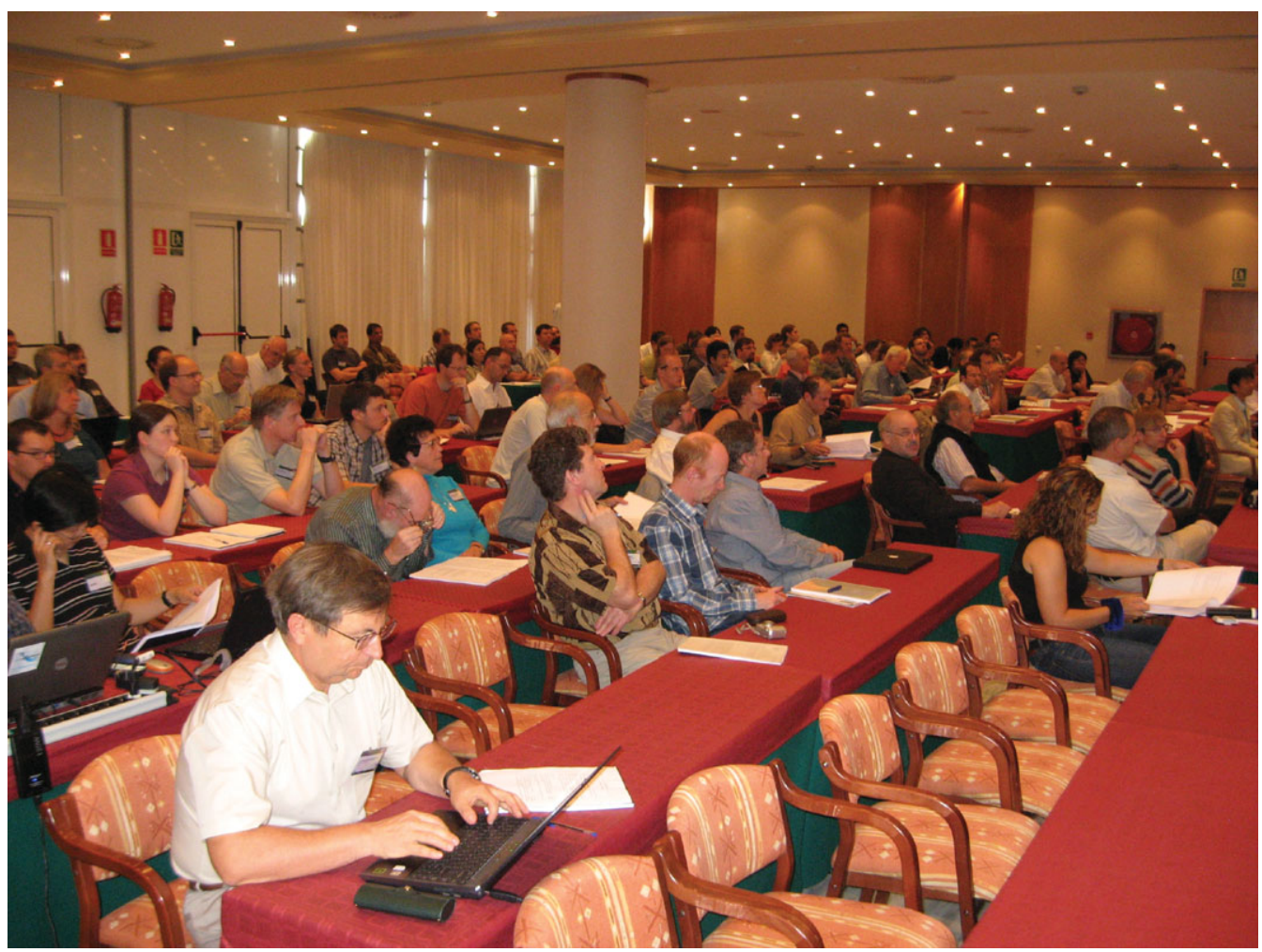

Inside the lecture room 


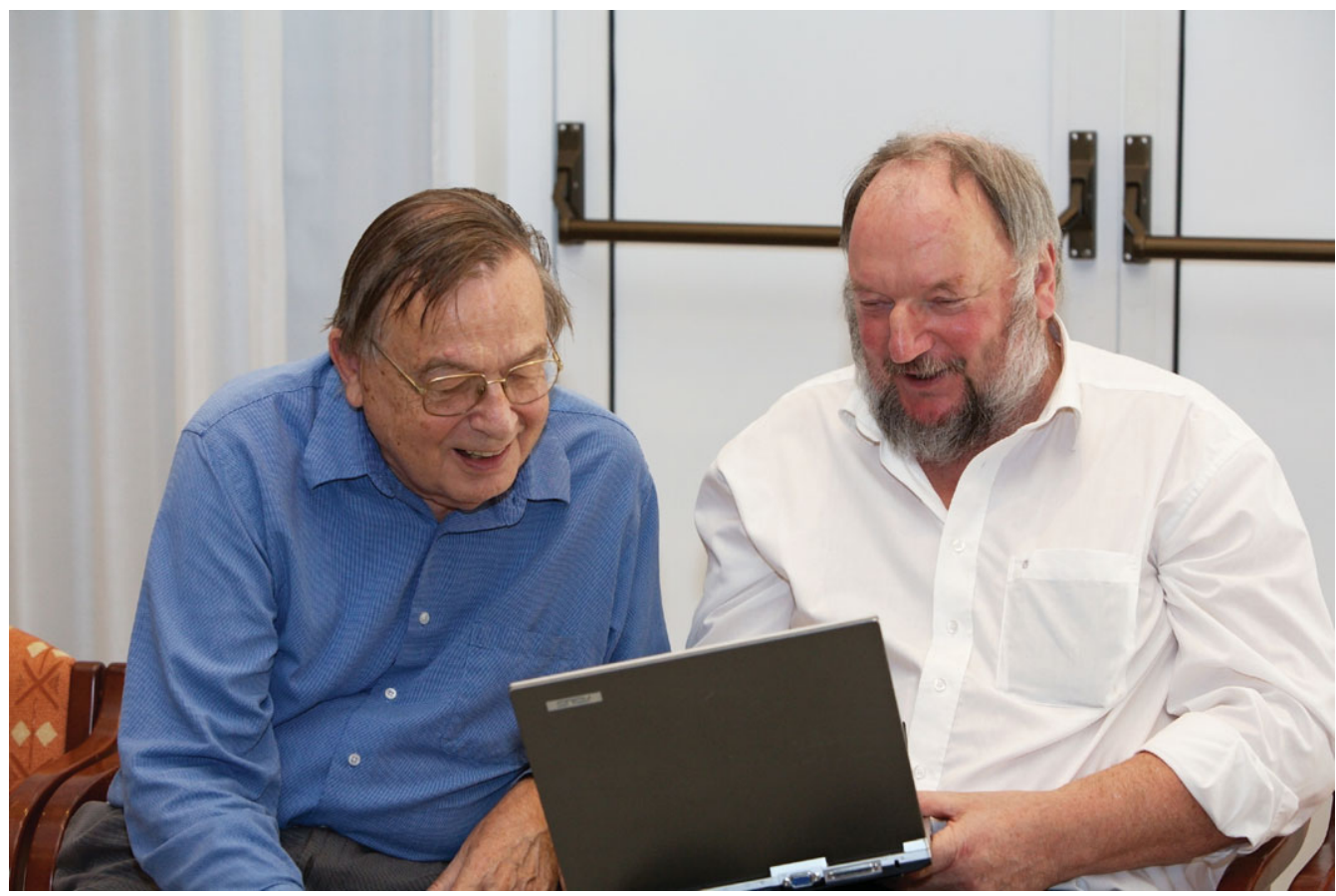

Enjoying common dynamo problems ... Paul Roberts (left) and Günther Rüdiger (right)

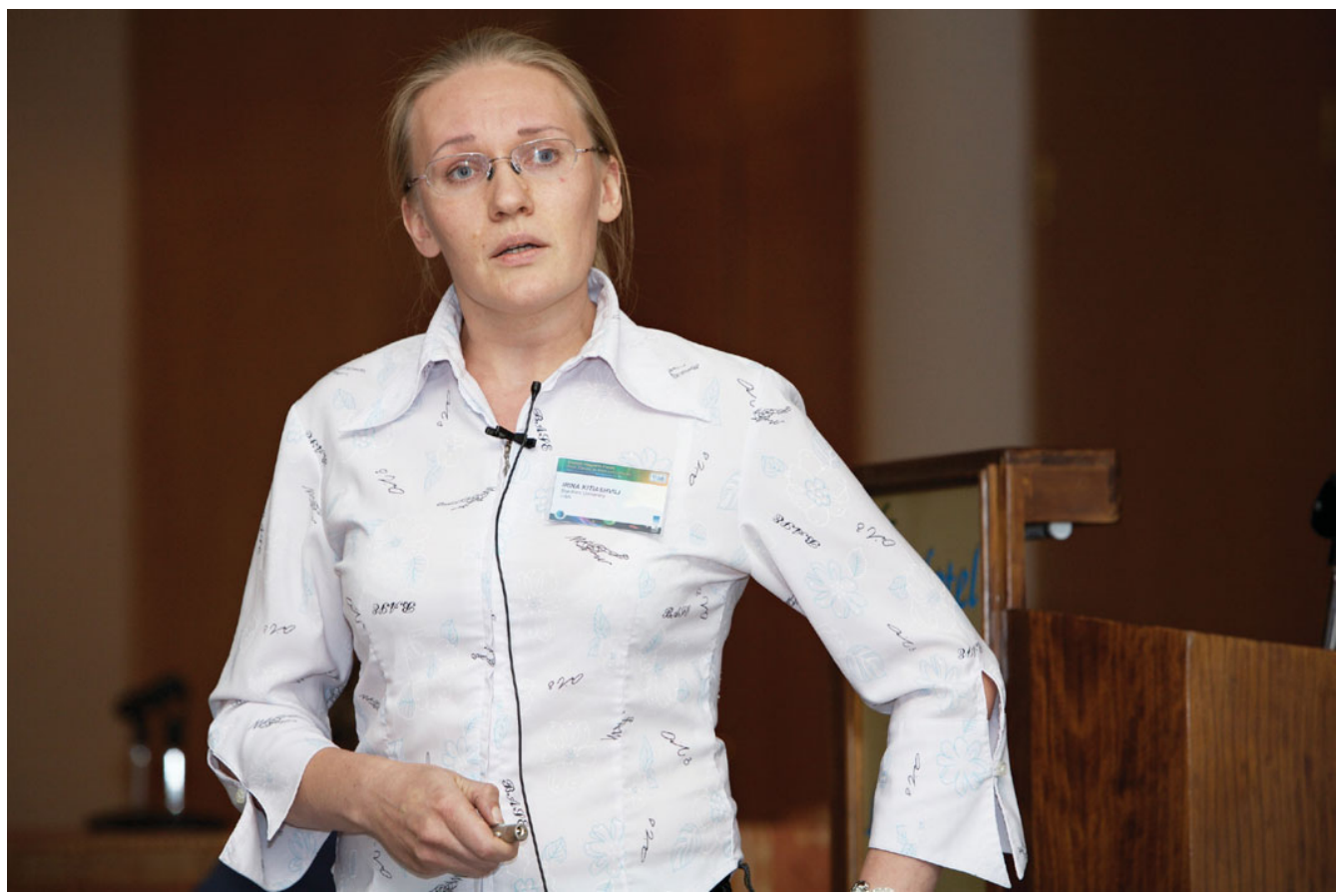

Irina Kitiashvili 